Article

\title{
Mechanisms for the Direct Electron Transfer of Cytochrome c Induced by Multi-Walled Carbon Nanotubes
}

\author{
Hua-Zhang Zhao ${ }^{1,2}$, Qian Du ${ }^{3}$, Zhen-Shan Li ${ }^{1,2}$ and Qin-Zheng Yang ${ }^{3, *}$
}

1 Department of Environmental Engineering, Peking University, Beijing 100871, China;

E-Mails: zhaohuazhang@pku.edu.cn (H.-Z.Z.); lizhenshan@iee.pku.edu.cn (Z.-S.L.)

2 The Key Laboratory of Water and Sediment Sciences, Ministry of Education, Beijing 100871, China

3 School of Food and Bioengineering, Shandong Institute of Light Industry, Jinan 250353, China; E-Mail: duqian0330@163.com

* Author to whom correspondence should be addressed; E-Mail: yangqinzheng@sina.com; Tel./Fax: +86-531-8801-1879.

Received: 18 June 2012; in revised form: 17 July 2012 / Accepted: 17 July 2012 /

Published: 2 August 2012

\begin{abstract}
Multi-walled carbon nanotube (MWCNT)-modified electrodes can promote the direct electron transfer (DET) of cytochrome c (Cyt c). There are several possible mechanisms that explain the DET of Cyt c. In this study, several experimental methods, including Fourier transform infrared spectroscopy, circular dichroism, ultraviolet-visible absorption spectroscopy, and electron paramagnetic resonance spectroscopy were utilized to investigate the conformational changes of Cyt $\mathrm{c}$ induced by MWCNTs. The DET mechanism was demonstrated at various nano-levels: secondary structure, spatial orientation, and spin state. In the presence of MWCNTs, the secondary structure of Cyt c changes, which exposes the active site, then, the orientation of the heme is optimized, revolving the exposed active center to the optimum spatial orientation for DET; and finally, a transition of spin states is induced, providing relatively high energy and a more open microenvironment for electron transfer. These changes at different nano-levels are closely connected and form a complex process that promotes the electron transfer of Cyt c.
\end{abstract}

Keywords: direct electron transfer (DET); cytochrome c (Cyt c); multi-walled carbon nanotube (MWCNT); secondary structure 


\section{Introduction}

Cytochrome c (Cyt c) is a water-soluble heme protein with a prosthetic iron porphyrin group. It is one of the major members of the biological respiratory chain and acts as an electron carrier, receiving electrons from Cyt $\mathrm{c}$ reductase and delivering them to Cyt $\mathrm{c}$ oxidase. Cyt $\mathrm{c}$ is usually used to modify electrodes to promote direct electron transfer (DET) for biosensors and biofuel cells [1-3]. However, the redox center of Cyt $\mathrm{c}$ is deeply embedded in the protein, and the protein is easily denatured upon adsorption onto a bare electrode surface, resulting in extremely slow electron-transfer kinetics and a poor response. To overcome these problems and promote fast electron transfer, electron mediators, such as Santa Barbara Amorphous (SBA-15), gold nanostructures, and carbon nanotubes (CNTs), have been used with Cyt $\mathrm{c}$ to modify substrate electrodes [4-8]. DET between Cyt $\mathrm{c}$ and electrodes has been observed with the help of the electron mediators.

The DET of Cyt $\mathrm{c}$ is a very complex process that has long been disputed. It is thought that the use of electron mediators overcomes some of the obstacles of DET. Electron mediators can provide a suitable surface at the electrode-solution interface, which can preserve the bioactivity of Cyt $\mathrm{c}$ and reduce the interfacial resistance between Cyt $\mathrm{c}$ and the electrode surface [4-11]. Partially unfolded structures of Cyt $\mathrm{c}$ and a shorter tunneling distance between Cyt $\mathrm{c}$ and the electrode are also induced by electron mediators [9].

Cyt c probably undergoes conformational changes during the electron transfer process [12]. Investigating these changes will provide insight into the DET mechanism. The secondary structure content changes when Cyt $\mathrm{c}$ is absorbed on a single-wall carbon nanotube - modified glassy carbon (GC) electrode [13]. These changes in secondary structure lead to heme exposure, which may influence DET $[14,15]$. The orientation and symmetry changes of the heme prophyrin ring on the electrode surface induced by electron mediators (such as SBA-15, gold nanostructures, and nitrogenous bases) also play an important role in DET $[4,8,16]$. In addition, changes in heme spin states affect the electronic activity of heme, which is usually closely related to electron transfer [17-20].

The above-mentioned studies used different electron mediators and focused on a single level of Cyt c (secondary structure, spatial orientation or spin state). It is not clear, however, whether the above-mentioned conformational changes coexist at different levels and how they relate to one another for a single electron mediator.

In recent decades, increasing attention has focused on CNTs due to their unique electronic properties and extremely high superficial volume ratio, which is useful for electron-transfer reactions. CNT-modified electrodes have been used in electrocatalytic reactions [21-23], and many studies have shown that DET can occur between CNTs and various biomolecules including Cyt c [5,7,24-26].

This paper aims to investigate the DET mechanism of Cyt $\mathrm{c}$ induced by electron mediators (for example, multi-walled carbon nanotubes, MWCNTs) at different levels. Cyclic voltammetry (CV), Fourier transform infrared (FTIR) spectroscopy, circular dichroism (CD), ultraviolet-visible (UV-vis) absorption spectroscopy, and electron paramagnetic resonance spectroscopy (EPR) were used to investigate DET and analyze the conformational changes of Cyt c. The DET mechanisms of Cyt c induced by MWCNTs were analyzed at different levels. 


\section{Experimental Section}

\subsection{Chemicals}

Horse heart Cyt c (MW 12384, Sigma, St. Louis, MO, USA) was used without further purification. MWCNTs were purchased from Shenzhen Nanotech Port (Shenzhen, China). Prior to use, the MWCNTs were pretreated by sonication in a mixture of concentrated sulfuric acid-nitric acid (3:1, v/v) for about $4 \mathrm{~h}$, neutralized and filtered with a Minipore membrane (pore size of $0.22 \mu \mathrm{m}$ in diameter), and dried at $60{ }^{\circ} \mathrm{C}$ overnight to obtain purified MWCNTs. Phosphate buffer solution (PBS, 0.1 M, pH 7.0) was prepared from $\mathrm{Na}_{2} \mathrm{HPO}_{4}$ and $\mathrm{NaH}_{2} \mathrm{PO}_{4}$ and always employed as a supporting electrolyte. All other chemicals were of analytical grade. All the solutions were prepared with doubly distilled water.

\subsection{Preparation of Cyt c/MWCNT Composites}

The preparation process of Cyt c/MWCNT composites is summarized as follows: $1 \mathrm{mg}$ of purified MWCNTs was dispersed in $1 \mathrm{~mL}$ PBS (0.1 M, pH 7.0) with the aid of $10 \mathrm{~min}$ of ultrasonication to give $1 \mathrm{mg} / \mathrm{mL}$ black suspension in which $10 \mathrm{mg}$ of Cyt $\mathrm{c}$ was added. The mixture was stored at $4{ }^{\circ} \mathrm{C}$ for $12 \mathrm{~h}$ followed by centrifugation at $18,000 \mathrm{r} / \mathrm{min}$ for $10 \mathrm{~min}$ and the removal of the supernatant to obtain Cyt c/MWCNT nanocomposites. The Cyt c/MWCNT composites were washed with distilled water to remove the loosely adsorbed Cyt $\mathrm{c}$ molecules and were dried under a frozen vacuum. The Cyt c/MWCNT composites were characterized by FTIR, CD, UV-vis and EPR spectroscopy.

\subsection{Preparation of Cyt c/MWCNT Modified GC Electrode}

The GC electrode ( $3 \mathrm{~mm}$ in diameter) was polished sequentially with slurries of 0.3 and $0.05 \mu \mathrm{m}$ alumina to mirror and washed with double-distilled water and ethanol in an ultrasonic bath for $1 \mathrm{~min}$. After treatment with acid, MWCNTs were dispersed with the aid of ultrasonic agitation in $10 \mathrm{~mL}$ of DMF to give a $1 \mathrm{mg} / \mathrm{mL}$ black suspension. The GC electrode was coated by casting $15 \mu \mathrm{L}$ of suspension of the MWCNTs and dried under an infrared lamp. The above steps were repeated, and an MWCNT-modified electrode was obtained.

Next, Cyt c (10 mg) was dissolved in PBS ( $1 \mathrm{~mL}, 0.1 \mathrm{M})$. The Cyt c solution $(20 \mu \mathrm{L})$ was sprayed and deposited onto the MWCNT-modified electrode surfaces and dried at room temperature for $4 \mathrm{~h}$ to obtain a Cyt c/MWCNT modified electrode. A Cyt c/MWCNT modified electrode was obtained and stored at room temperature after $5 \mu \mathrm{L}$ of $0.5 \%$ Nafion solution was cast onto the modified electrode surfaces.

Prior to use, the Cyt c/MWCNT modified GC electrode and the bare GC electrode were activated using consecutive cyclic potential scans performed in $0.1 \mathrm{M} \mathrm{PBS}(\mathrm{pH} 6.24)$ for $1.5 \mathrm{~min}$ (potential range from 1.5 to $-1.0 \mathrm{~V}$, scan rate of $1 \mathrm{~V} / \mathrm{s}$ ).

\subsection{Characterization and Electrochemical Measurements}

Fourier transform infrared (FTIR) spectra made in ATR mode were recorded using a NICOLET iN10 MX FT-IR spectrophotometer (Thermo Scientific, West Palm Beach, FL, USA) to characterize the functionalized MWCNTs and Cyt c/MWCNT nanocomposites in dry state. For each sample, a total of 128 scans at a resolution of $4 \mathrm{~cm}^{-1}$ were collected. To investigate the changes in secondary structures of 
Cyt c immobilized on MWCNTs, the Gaussian curve-fitting method conducted by PeakFit 7.2 software was used to deconvolute the FTIR spectra and Origin 6.0 software was used to calculate the ratios of various secondary structure elements according to their integrated areas.

CD spectra were recorded from 200 to $240 \mathrm{~nm}$ with a $0.5 \mathrm{~s}$ response and $20 \mathrm{~nm} / \mathrm{min}$ scanning speed on a JASCO J-715 (Tokyo, Japan) spectropolarimeter using solution with protein concentrations of about $0.3 \mathrm{mg} / \mathrm{mL}$ for far-UV regions. The spectra were collected and averaged over 3 scans, using quartz cells of $1.0 \mathrm{~mm}$ optical path length. The results are expressed as molar ellipticity, $[\theta]\left(\mathrm{deg} \cdot \mathrm{cm}^{2} \cdot \mathrm{dmol}^{-1}\right)$. According to the Yang-Chen formula [27], the ratios of the second ary structure elements (a-helix, b-sheet, turns, and random coils) were calculated using the software package J-715 for Windows Secondary Structure Estimation (Version 1.0).

UV-vis absorption spectra were collected on a BWS003 UV-vis transmittance/reflectance spectrophotometer (B\&W Tek Inc., Newark, DE, USA). The UV-vis absorption spectrum of the solution was recorded by an Agilent 8453 UV-vis near-infrared spectrophotometer (Agilent Instruments, Englewood, CO, USA).

EPR spectra were recorded on a Bruker EMX spectrometer (Rheinstetten, Germany). The conditions for EPR measurements were as follows: frequency, $9.6 \mathrm{GHz}$; power, $3 \mathrm{~mW}$; modulation amplitude, $10 \mathrm{G}$; modulation frequency, $100 \mathrm{kHz}$; and temperature, $293 \mathrm{~K}$. As the short time stability of the magnetic field is $5 \mathrm{mG}$, all the $g$ factors have a maximum standard deviation of \pm 0.17 . The high-spin signal at $g=6$ was quantified by double integration with the lower integration limit taken below the low-fiby end of the spectrum and the upper limit at a field corresponding to a $g$ value of 4.67 .

\section{Results and Discussion}

\subsection{Direct Electrochemistry of Cyt c on the MWCNT-Modified GC Electrode}

CV experiments were conducted to clarify the DET between Cyt $\mathrm{c}$ and the MWCNT-modified electrode in PBS. As shown in Figure 1(A), a pair of well-defined redox peaks appeared at the MWCNT-modified GC electrode with the formal potential of $46 \mathrm{mV}$, which is almost in agreement with the reported formal potential of Cyt $\mathrm{c}$ on SWCNTs [13]. However, no peaks were apparent at the bare GC electrode. This suggests that the thin layer of MWCNT on the GC electrode assists in the DET from the active site of Cyt $\mathrm{c}$ to the electrode and that, in the absence of the MWCNTs, no electron transfer occurs at the electrode. This conclusion is consistent with other results of CNT-modified electrodes [28-33].

Figure 1(B,C) illustrates the CVs of Cyt $\mathrm{c}$ on the MWCNT-modified GC electrode at various scan rates. The anodic and cathodic peak currents changed linearly with the scan rate in the range of 200 to $1,000 \mathrm{mV} / \mathrm{s}$ (The correlation coefficients for the anodicand cathodic peak currents are both greater than 0.9997), while $I_{p} v s . v^{1 / 2}$ curves show an upward inclination, indicating that the electrode process is controlled by a surface-bound species [34,35]. Furthermore, the peak separations ranging from $135 \mathrm{mV}$ at a scan rate of $200 \mathrm{mV} / \mathrm{s}$ to $267 \mathrm{mV}$ at $1,000 \mathrm{mV} / \mathrm{s}$ indicate that the adsorbed Cyt c displayed a quasi-reversible electron-transfer process on MWCNTs. 
Figure 1. Cyclic voltammograms corresponding to (A) Cyt $\mathrm{c}$ with and without MWCNTs (scan rate: $50 \mathrm{mV} / \mathrm{s}$ ); (B) Cyt c on the MWCNT-modified GC electrode at various scan rates: $200,400,600,800$, and $1,000 \mathrm{mV} / \mathrm{s}$. (C) The dependence of (a) the anodic peak currents and (b) the cathodic peak currents on the scan rates and square root of scan rates. The activation potential window of the electrode is $1.5 \mathrm{~V}-1.0 \mathrm{~V}$. The supporting electrolyte was $1 \mathrm{M}$ PBS (pH 6.07) containing $0.1 \mathrm{M} \mathrm{NaCl}$.
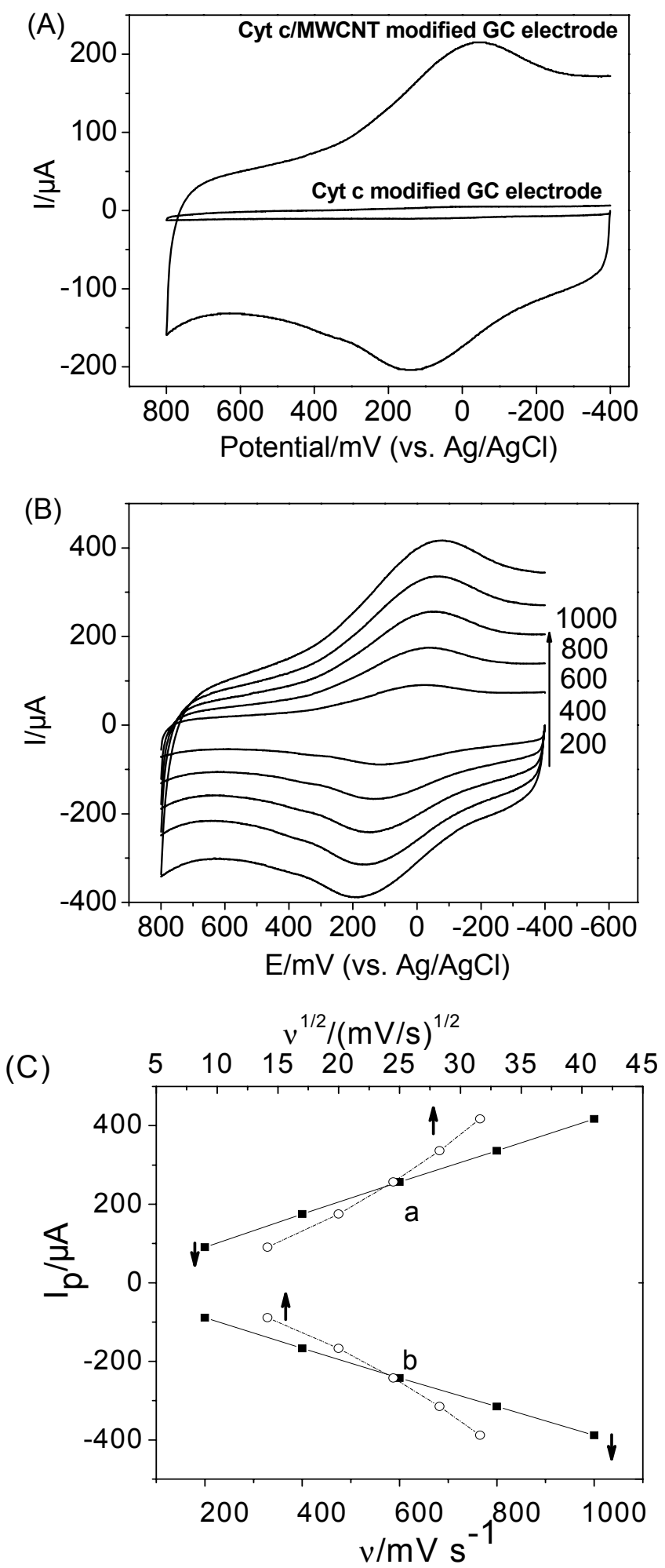


\subsection{FTIR Spectroscopy}

FT-IR spectroscopy was utilized to investigate the environmental and structural changes of Cyt c immobilized on MWCNTs to understand the molecular reaction mechanism. The FTIR spectra of MWCNTs, Cyt c, and Cyt c/MWCNTs are shown in Figure 2.

Figure 2. FTIR spectra of MWCNTs, Cyt c, and Cyt c/MWCNTs.

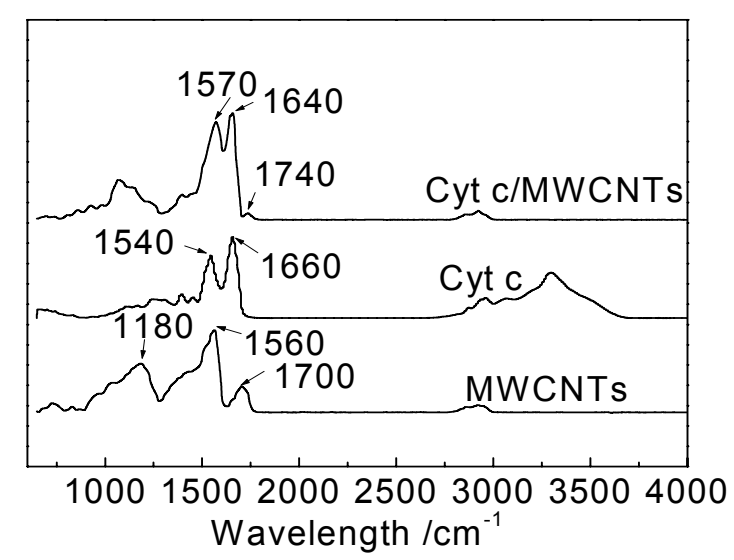

For the MWCNTs after acidification, the peaks at 1,700 and $1,560 \mathrm{~cm}^{-1}$ in the IR spectrum of MWCNTs suggest the presence of carboxyl and carboxylate groups, respectively, while the peak at $1,180 \mathrm{~cm}^{-1}$ corresponds to the $\mathrm{C}-\mathrm{O}$ bond. The FTIR spectrum of Cyt $\mathrm{c}$ shows two characteristic adsorption peaks at $1,660 \mathrm{~cm}^{-1}$ and $1,540 \mathrm{~cm}^{-1}$, corresponding to the amide I and amide II adsorption bands of protein molecules, respectively $[4,36]$. The amide I vibration is caused by the $\mathrm{C}=\mathrm{O}$ stretching vibration of peptide linkages in the protein's backbone, and the amide II vibration results from a combination of N-H in-plane bending and C-N stretching vibrations of the peptide groups. They are widely used to monitor changes in secondary structure. The FTIR spectrum of Cyt c immobilized on MWCNTs also shows amide I and amide II adsorption bands, but they shift to some extent. The amide I maximum shifts to lower frequencies from 1,660 to $1,640 \mathrm{~cm}^{-1}$, and the amide II maximum shifts to higher frequencies from 1,540 to $1,570 \mathrm{~cm}^{-1}$, while the small shift values also indicate that Cyt c retains its natural biological activity.

The slight shift of the amide I and amide II bands of Cyt $\mathrm{c}$ absorbed on MWCNTs indicates a corresponding change in secondary structure. Deconvolution of the amide I band of Cyt c before and after absorption onto MWCNTs followed by curve fitting with a Gaussian function yields several distinct underlying absorption bands shown in Figure 3.

Figure 3(A) depicts the spectral decomposition of amide I for Cyt c. The bands at 1,618 was assigned to side chain interactions not associated with the secondary structure [37]. The $1,639 \mathrm{~cm}^{-1}$ peak was assigned to beta-sheet structures, which made up $21 \%$ of the total area of the amide I band of Cyt c. Alpha-helical structures are characterized by a band at around 1,659 $\mathrm{cm}^{-1}$; this component accounts for $36 \%$ of the total area of the amide I band. Bands at $1,647 \mathrm{~cm}^{-1}$ and $1,678 \mathrm{~cm}^{-1}$ correspond to random structures and turns, respectively. These structures make up $13 \%$ and $25 \%$ of the protein, respectively.

Figure 3(B) illustrates the spectral decomposition of amide I for Cyt c immobilized on MWCNTs. Compared with Figure 3(A), the alpha-helix peak position shifts from $1,659 \mathrm{~cm}^{-1}$ to $1,661 \mathrm{~cm}^{-1}$ while 
the peaks for the beta-sheet structures almost unchanged. The alpha-helical content decreases from $36 \%$ to $33 \%$ while the beta-sheet content increases from $21 \%$ to $26 \%$. These data suggest that the interaction between Cyt $\mathrm{c}$ and MWCNTs results in the conversion of alpha-helices to beta-sheets.

Figure 3. Amide I band peak fitting in the original FTIR spectra for Cyt c (A) and Cyt c/MWCNTs (B). The original envelope ( $\square$ ), the component bands (dashed line), and the generated envelope (solid line) are shown, respectively.
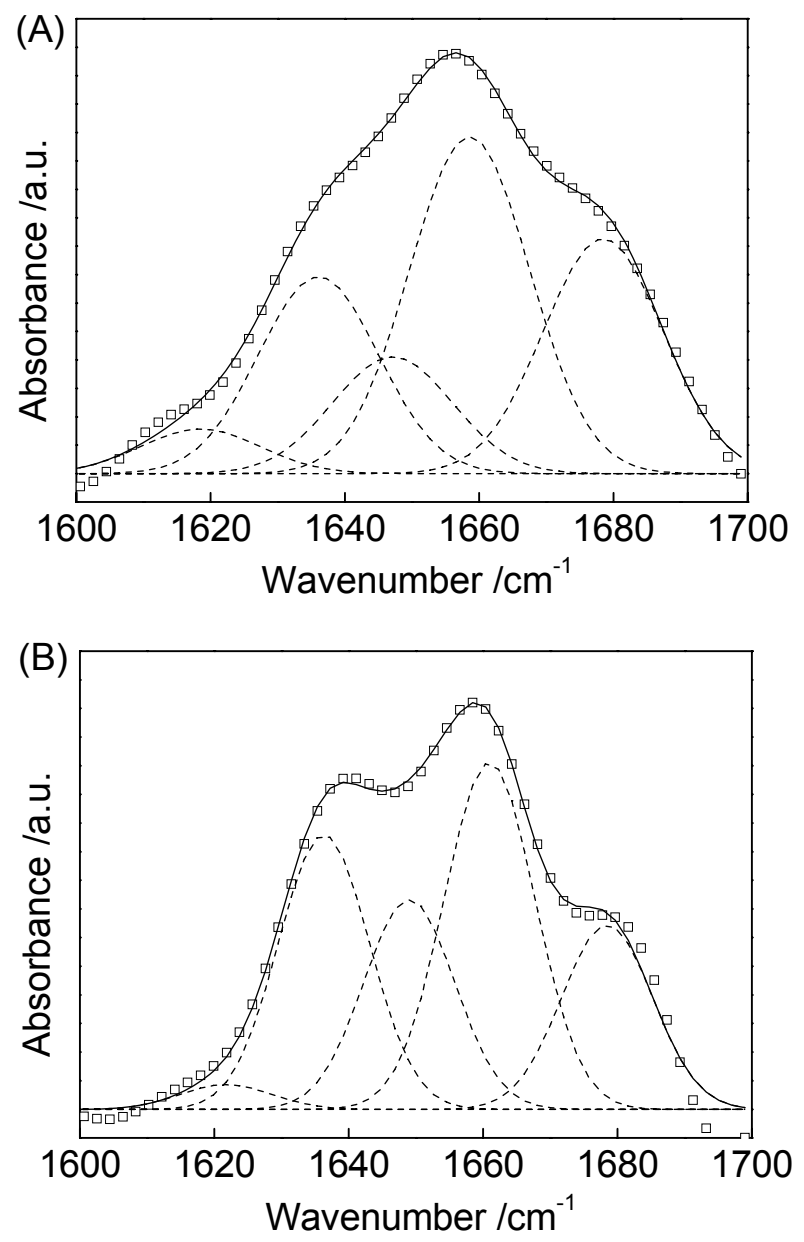

\subsection{Spectroscopy}

The FTIR spectra focus on the changes in functional groups, and they provide preliminary conformational analyses of solid-state proteins. $\mathrm{CD}$ is an advanced tool to investigate the conformational changes of proteins in a dilute aqueous solution. In the present work, CD was applied to give an overview of the secondary structures of Cyt $\mathrm{c}$ interacting with MWCNTs.

The far-UV CD spectra of native Cyt $\mathrm{c}$ and Cyt $\mathrm{c}$ immobilized on MWCNTs exhibit two negative peaks at 208 and $221 \mathrm{~nm}$ resulting from the chirality of alpha-helical structures (Figure 4). These results are similar to those of previous reports [38,39]. Absorption in the region below $240 \mathrm{~nm}$ is due primarily to the peptide bond. Therefore, it can be concluded from the CD spectra that the structure and activity of Cyt $\mathrm{c}$ after being treated with MWCNTs are essentially the same as in the native protein. The secondary structure content of the native and the treated Cyt $\mathrm{c}$ were calculated and the results are compared with the corresponding results of FTIR spectra as follows. 
Figure 4. Far-UV CD spectra of Cyt c and Cyt c/MWCNTs.

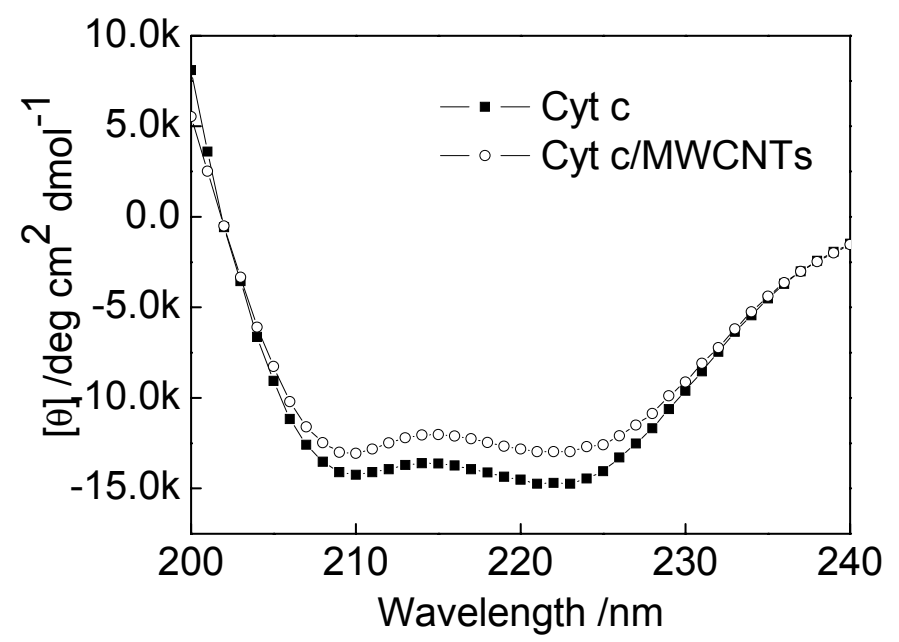

Both FTIR and CD spectra reveal a decrease in alpha-helices (FTIR: from 36\% to 33\%; CD: from $26 \%$ to $23 \%$ ) and an increase in beta-sheets (FTIR: from 21\% to 26\%; CD: from $12 \%$ to 13\%). These results further confirmed that the presence of MWCNTs cause a decrease in alpha-helices in Cyt c.

The alpha-helices are mainly located on the exterior of the protein and are more compact than other secondary structures. As a result, the alpha-helical structure is one of the barriers for electron transfer [33]. Reducing the alpha-helical content loosens the protein shell and exposes the active site. Thus, it is easier to approach the active center, facilitating DET.

\subsection{UV-Vis Absorption Spectroscopy}

The spatial orientation of the heme porphyrin ring, which may be a factor that affects electron transfer, was investigated by UV-vis absorption spectroscopy, as shown in Figure 5. There is no absorption observed in Figure 5 for the black MWCNT particles. The spectrum of Cyt c exhibits two obvious UV absorption peaks at 409 and $528 \mathrm{~nm}$, corresponding to the Soret band and Q-band of Cyt c, respectively. The spectrum of Cyt $\mathrm{c}$ immobilized on MWCNTs also shows a Soret band with a decrease in intensity and a hypsochromic shift of only $1 \mathrm{~nm}$ to $408 \mathrm{~nm}$.

Figure 5. UV-vis absorption spectra of MWCNTs, Cyt c, and Cyt c/MWCNTs.

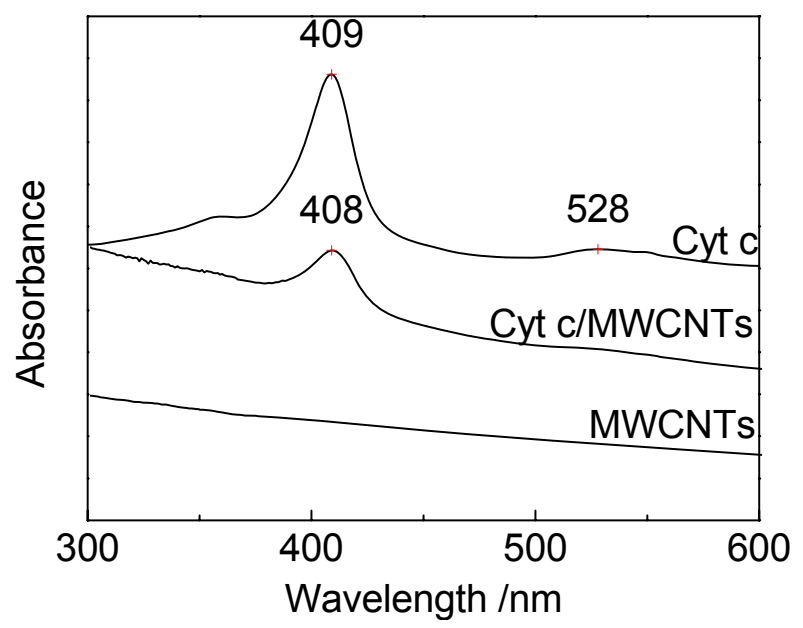


The Soret band results from the additive effects of the transition dipole moments of the two orbital excitations $a_{1 u}-e_{g}$ and $a_{2 u}-e_{g}$ of $\pi-\pi *$ transitions of the porphyrin ring in Cyt $c$; therefore, the intensity of the Soret band reflects the symmetry of the porphyrin ring (the spatial orientation) $[4,13,39,40]$, and the shift of the Soret band reflects the conformational changes of the heme microenvironment $[4,41]$.

For Cyt c immobilized on MWCNTs, the small shift of the Soret band suggests that the interaction between Cyt $\mathrm{c}$ and MWCNTs has not destroyed the biological activity of Cyt $\mathrm{c}$, but has changed its microstructure. These results agree with previous studies on Cyt $\mathrm{c}$ absorbed on SBA-15 and gold nanostructures $[4,8,42]$. It is suspected that some intermediate states of the heme porphyrin ring are produced during the electron transfer process, and the $\pi-\pi^{*}$ transition is decreased [16]. As a result, the intensity of the Soret band decreases as shown in Figure 5. As the dipole direction is consistent with the spatial orientation [43], these intermediate states may correspond to some transient spatial orientations of the heme porphyrin ring, which are characterized by the changes in the symmetry of the porphyrin ring. The transient spatial orientations probably facilitate the electron transfer of Cyt c $[4,44]$. As suggested above, it is possible that the MWCNT surface provides a more favorable spatial orientation of the heme porphyrin ring for DET.

From another point of view, the shift and the intensity decrease of the Soret band may result from the decrease in the hydrophobic nature of Cyt $\mathrm{c}$. The hydrophobic nature is closely related to the amount of alpha-helices due to their compactness and specific location in the protein exterior [4,15]. The FTIR and CD results indicate that the alpha-helical content decreases when Cyt $\mathrm{c}$ interacts with MWCNTs, which results in a decrease in the hydrophobic nature of Cyt $\mathrm{c}$. The protein orientation may also be optimized by a decrease in hydrophobic interactions [4], and DET would be enhanced with the appropriate orientation.

\subsection{EPR Spectroscopy}

As has been reported, the spin state equilibrium of the heme iron could modulate both substrate binding and the oxidation-reduction reactions of the cytochrome [19], and electron transfer is always accompanied by a change in iron spin state [20]. We speculate that the spin state influences the electron transfer activity. EPR spectra were utilized to investigate the conversion of the spin states, and the heme iron axial ligation is shown in Figure 6. As shown in Figure 6, the signals of the native Cyt c represent a mixture of high and low spin states, characterized at $g=6.19$ and $g=4.36$, respectively [17]. The signal at $\mathrm{g}=1.98$ is from the strong Fe-S ligation in the heme redox center. Fe-S ligation is important for the bioactivity of Cyt c, and damage to the Fe-S ligation will cause deactivation of the protein. When Cyt c interacts with MWCNTs, the Fe-S ligation signal nearly stay the same, suggesting that $\mathrm{Fe}-\mathrm{S}$ ligation has not been destroyed, and the protein retains its bioactivity in this process. The low spin signal has a red shift to $g=4.45$ along with a decrease in intensity while the high spin signal is observed at $\mathrm{g}=6.26$ along with an increase in intensity. This result suggests that the interaction between Cyt $\mathrm{c}$ and MWCNTs induces a transition of the heme iron from a low spin state to a high spin state.Cyt c has six heme iron coordination sites [45]. Low spin hemes are six-coordinated, which means that the six coordination sites of the heme iron are occupied by intrinsic ligands. In contrast, high spin heme compounds are formally five-coordinated, leaving a coordination site open for the binding of extrinsic ligands [19]. X-ray structures of model compounds and proteins have shown that the high-spin iron atom sits about $0.5 \AA$ out of the porphyrin plane while the low-spin iron is 
in-plane [46,47]. Accordingly, high spin states have a relatively high energy and provide a more open microenvironment for electron transfer than low spin states. According to the observed transition to high spin states as shown in Figure 6, the active center of the heme ring is in a state that facilitates DET. In addition, the transition of the spin states is accompanied by significant nuclear reorganization [20] to optimize the orientation of the heme ring for DET, which is similar to the results from the UV-vis spectroscopy.

Figure 6. EPR spectra of Cyt $\mathrm{c}$ and Cyt c/MWCNTs.

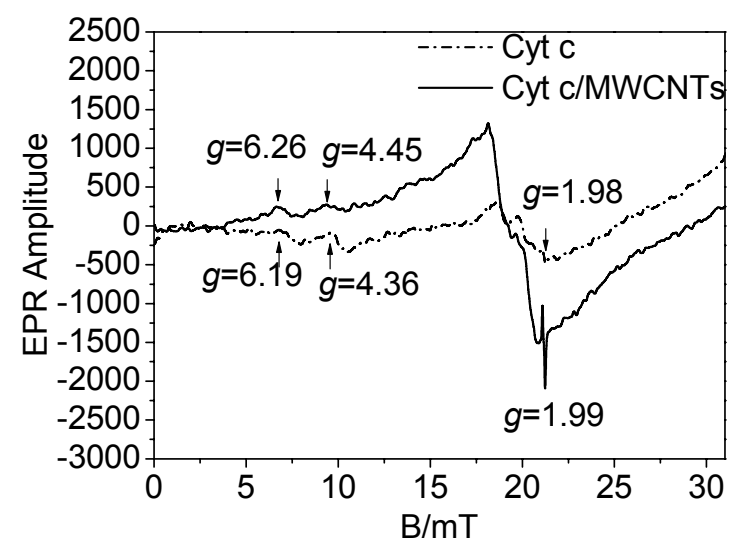

The above assays confirm that Cyt $\mathrm{c}$ goes through the above conformational changes at three levels: secondary structure, spatial orientation, and spin states. The changes in the three levels are not isolated, but closely interrelated; changes in secondary structure provide an exposed active center; changes in the spatial orientation of the porphyrin ring position the active center in a more favorable orientation to receive and transmit electrons; changes in spin states make the active center more appropriate for high electronic activity. As a result, a microenvironment with an open enzyme active center, the correct orientation of the active center, and an appropriate state for the electrons is created by which DET between Cyt $\mathrm{c}$ and MWCNT-modified electrodes is achieved and enhanced.

\section{Conclusions}

DET between Cyt $\mathrm{c}$ and MWCNT-modified electrodes was achieved in this work. The conformational changes of Cyt $\mathrm{c}$ at different nano-levels were investigated using different analytical methods (FITR, CD, UV, and EPR) to study the DET mechanism. First, MWCNTs affect the ratio of secondary structure elements, leading to exposure of the active site. Then, the exposed active center is oriented in the correct orientation for DET. Finally, a transition of spin states is induced to provide relatively high energy and a more open microenvironment for electron transfer. The DET mechanism could apply to other electron mediators and proteins. The understanding of the mechanism would contribute to the development of modified electrodes and biosensors.

\section{Acknowledgments}

The authors are grateful for the financial support from the National Natural Science Fund (Grant No. 21077001) and the National Twelfth Five-Year Technology Support Program (Grant No. 2011BAJ07B04) of China. 


\section{References}

1. Dai, Z.H.; Liu, S.Q.; Ju, H.X. Direct electron transfer of cytochrome c immobilized on a nay zeolite matrix and its application in biosensing. Electrochim. Acta 2004, 49, 2139-2144.

2. Ramanavicius, A.; Ramanaviciene, A. Hemoproteins in design of biofuel cells. Fuel Cells 2009, 9 , $25-36$.

3. Gobi, K.V.; Mizutani, F. Efficient mediatorless superoxide sensors using cytochrome c-modified electrodes: Surface nano-organization for selectivity and controlled peroxidase activity. J. Electroanal. Chem. 2000, 484, 172-181.

4. Liu, H.Q.; Tian, Y.; Deng, Z.F. Morphology-Dependent electrochemistry and electrocatalytical activity of cytochrome c. Langmuir 2007, 23, 9487-9494.

5. Wang, J.X.; Li, M.X.; Shi, Z.J.; Li, N.Q.; Gu, Z.N. Direct electrochemistry of cytochrome c at a glassy carbon electrode modified with single-wall carbon nanotubes. Anal. Chem. 2002, 74, 1993-1997.

6. Yin, Y.J.; Wu, P.; Lu, Y.F.; Du, P.; Shi, Y.M.; Cai, C.X. Immobilization and direct electrochemistry of cytochrome c at a single-walled carbon nanotube-modified electrode. J. Solid State Electrochem. 2007, 11, 390-397.

7. Zhao, G.C.; Yin, Z.Z.; Zhang, L.; Wei, X.W. Direct electrochemistry of cytochrome c on a multi-walled carbon nanotubes modified electrode and its electrocatalytic activity for the reduction of $\mathrm{h}_{2} \mathrm{O}_{2}$. Electrochem. Commun. 2005, 7, 256-260.

8. Zhu, L.; Wang, K.Q.; Lu, T.H.; Xing, W.; Li, J.; Yang, X.G. The direct electrochemistry behavior of cyt $\mathrm{c}$ on the modified glassy carbon electrode by sba-15 with a high-redox potential. $J$. Mol. Catal. B: Enzym. 2008, 55, 93-98.

9. Caban, K.; Offenhausser, A.; Mayer, D. Electrochemical characterization of the effect of gold nanoparticles on the electron transfer of cytochrome c. Phys. Status Solidi A-Appl. Mat. 2009, 206, 489-500.

10. Eddowes, M.J.; Hill, H.A.O.; Uosaki, K. Electrochemistry of cytochrome-c: Comparison of the electron-transfer at a surface-modified gold electrode with that to cytochrome-oxidase. J. Am. Chem. Soc. 1979, 101, 7113-7114.

11. Peng, L.; You, S.J.; Wang, J.Y. Carbon nanotubes as electrode modifier promoting direct electron transfer from shewanella oneidensis. Biosens. Bioelectron. 2010, 25, 1248-1251.

12. El Kasmi, A.; Leopold, M.C.; Galligan, R.; Robertson, R.T.; Saavedra, S.S.; El Kacemi, K.; Bowden, E.F. Adsorptive immobilization of cytochrome $\mathrm{c}$ on indium/tin oxide (ito): Electrochemical evidence for electron transfer-induced conformational changes. Electrochem. Commun. 2002, 4, 177-181.

13. Jiang, X.; Zhang, Z.L.; Bai, H.Y.; Qu, X.H.; Jiang, J.G.; Wang, E.K.; Dong, S.J. Effect of electrode surface microstructure on electron transfer induced conformation changes in cytochrome $\mathrm{c}$ monitored by in situ uv and cd spectroelectrochemistry. Spectroc. Acta Pt. A-Mol. Biomol. Spectr. 2005, 61, 943-951.

14. Balakrishnan, G.; Hu, Y.; Oyerinde, O.F.; Su, J.; Groves, J.T.; Spiro, T.G. A conformational switch to beta-sheet structure in cytochrome c leads to heme exposure. Implications for cardiolipin peroxidation and apoptosis. J. Am. Chem. Soc. 2007, 129, 504-505. 
15. Cortese, J.D.; Voglino, A.L.; Hackenbrock, C.R. Multiple conformations of physiological membrane-bound cytochrome c. Biochemistry 1998, 37, 6402-6409.

16. Wu, L.L.; Huang, H.G.; Li, J.X.; Luo, J.; Lin, Z.H. Time-resolved uv-vis spectroelectrochemical studies of the electron transfer process of cytochrome c. Electrochim. Acta 2000, 45, 2877-2881.

17. Bagel'ova, J.; Fedunova, D.; Gazova, Z.; Fabian, M.; Antalik, M. Influence of nacl and sorbitol on the stability of conformations of cytochrome c. Biophys. Chem. 2008, 135, 110-115.

18. Jimenez, H.R.; de Sola, E.R.; Moratal, J.M.; Arbona, M. Protein unfolding: H-1-nmr studies of paramagnetic ferricytochrome c-550 from horse heart. Z. Anorg. Allg. Chem. 2005, 631, 2020-2025.

19. Sligar, S.G. Coupling of spin, substrate, and redox equilibria in cytochrome p450. Biochemistry 1976, 15, 5399-5406.

20. Turner, J.W.; Schultz, F.A. Coupled electron-transfer and spin-exchange reactions. Coord. Chem. Rev. 2001, 219, 81-97.

21. Davis, J.J.; Coles, R.J.; Hill, H.A.O. Protein electrochemistry at carbon nanotube electrodes. J. Electroanal. Chem. 1997, 440, 279-282.

22. Sun, J.J.; Zhao, H.Z.; Yang, Q.Z.; Song, J.; Xue, A. A novel layer-by-layer self-assembled carbon nanotube-based anode: Preparation, characterization, and application in microbial fuel cell. Electrochim. Acta 2010, 55, 3041-3047.

23. Zhao, G.; Liu, K.Z.; Lin, S.; Liang, J.; Guo, X.Y.; Zhang, Z.J. Electrocatalytic reduction of nitrite using a carbon nanotube electrode in the presence of cupric ions. Microchim. Acta 2004, 144, $75-80$.

24. Gooding, J.J.; Wibowo, R.; Liu, J.Q.; Yang, W.R.; Losic, D.; Orbons, S.; Mearns, F.J.; Shapter, J.G.; Hibbert, D.B. Protein electrochemistry using aligned carbon nanotube arrays. J. Am. Chem. Soc. 2003, 125, 9006-9007.

25. Vaze, A.; Hussain, N.; Tang, C.; Leech, D.; Rusling, J. Biocatalytic anode for glucose oxidation utilizing carbon nanotubes for direct electron transfer with glucose oxidase. Electrochem. Commun. 2009, 11, 2004-2007.

26. Zheng, W.; Zhao, H.Y.; Zhou, H.M.; Xu, X.X.; Ding, M.H.; Zheng, Y.F. Electrochemistry of bilirubin oxidase at carbon nanotubes. J. Solid State Electrochem. 2010, 14, 249-254.

27. Chen, Y.H.; Yang, J.T.; Martinez, H.M. Determination of secondary structures of proteins by circular-dichroism and optical rotatory dispersion. Biochemistry 1972, 11, 4120-4131.

28. Cai, C.X.; Chen, J. Direct electrochemistry of horseradish peroxidase at a carbon nanotube electrode. Acta Chim. Sinica 2004, 62, 335-340.

29. Guiseppi-Elie, A.; Lei, C.H.; Baughman, R.H. Direct electron transfer of glucose oxidase on carbon nanotubes. Nanotechnology 2002, 13, 559-564.

30. Liu, J.Q.; Chou, A.; Rahmat, W.; Paddon-Row, M.N.; Gooding, J.J. Achieving direct electrical connection to glucose oxidase using aligned single walled carbon nanotube arrays. Electroanalysis 2005, 17, 38-46.

31. Lu, Y.F.; Yin, Y.J.; Wu, P.; Cai, C.X. Direct electrochemistry and bioelectrocatalysis of myoglobin at a carbon nanotube-modified electrode. Acta Phys.-Chim. Sin. 2007, 23, 5-11. 
32. Salimi, A.; Noorbakhsh, A.; Ghadermarz, M. Direct electrochemistry and electrocatalytic activity of catalase incorporated onto multiwall carbon nanotubes-modified glassy carbon electrode. Anal. Biochem. 2005, 344, 16-24.

33. Zhao, H.Z.; Sun, J.J.; Song, J.; Yang, Q.Z. Direct electron transfer and conformational change of glucose oxidase on carbon nanotube-based electrodes. Carbon 2010, 48, 1508-1514.

34. Li, Q.L.; Chen, S.U. Studies on electrochemical-behavior of cephalexin. Anal. Chim. Acta 1993, $282,145-152$.

35. Cao, W.; Wei, C.M.; Hu, J.B.; Li, Q.L. Direct electrochemistry and electrocatalysis of myoglobin immobilized on gold nanoparticles/carbon nanotubes nanohybrid film. Electroanalysis 2008, 20, 1925-1931.

36. Jackson, M.; Mantsch, H.H. The use and misuse of ftir spectroscopy in the determination of protein-structure. Crit. Rev. Biochem. Mol. Biol. 1995, 30, 95-120.

37. Speare, J.O.; Rush, T.S. Ir spectra of cytochrome c denatured with deuterated guanidine hydrochloride show increase in beta sheet. Biopolymers 2003, 72, 193-204.

38. Jiang, X.; Qu, X.H.; Zhang, L.; Zhang, Z.L.; Jiang, J.G.; Wang, E.K.; Dong, S.J. Ph-dependent conformational changes of ferricytochrome $\mathrm{c}$ induced by electrode surface microstructure. Biophys. Chem. 2004, 110, 203-211.

39. Hagarman, A.; Duitch, L.; Schweitzer-Stenner, R. The conformational manifold of ferricytochrome c explored by visible and far-uv electronic circular dichroism spectroscopy. Biochemistry 2008, 47, 9667-9677.

40. Hawkridge, F.M.; Taniguchi, I. The direct electron-transfer reactions of cytochrome-c at electrode surfaces. Comments Inorg. Chem. 1995, 17, 163-187.

41. George, P.; Hanania, G. A spectrophotometric study of ionizations in methaemoglobin. Biochem. J. 1953, 55, 236-243.

42. Feng, J.J.; Zhao, G.; Xu, J.J.; Chen, H.Y. Direct electrochemistry and electrocatalysis of heme proteins immobilized on gold nanoparticles stabilized by chitosan. Anal. Biochem. 2005, 342, 280-286.

43. Kuznetsov, B.A.; Byzova, N.A.; Shumakovich, G.P. The effect of the orientation of cytochrome-c molecules covalently attached to the electrode surface upon their electrochemical activity. J. Electroanal. Chem. 1994, 371, 85-92.

44. Nikiforov, M.P.; Zerweck, U.; Milde, P.; Loppacher, C.; Park, T.H.; Uyeda, H.T.; Therien, M.J.; Eng, L.; Bonnell, D. The effect of molecular orientation on the potential of porphyrin-metal contacts. Nano Lett. 2008, 8, 110-113.

45. Doussiere, J.; Gaillard, J.; Vignais, P.V. Electron transfer across the $\mathrm{o}_{2}{ }^{-}$generating flavocytochrome $\mathrm{b}$ of neutrophils. Evidence for a transition from a low-spin state to a high-spin state of the heme iron component. Biochemistry 1996, 35, 13400-13410.

46. Hoard, J.L. Stereochemistry of hemes and other metalloporphyrins. Science 1971, 174, 1295-1302.

47. Perutz, M.F. Stereochemistry of cooperative effects in haemoglobin. Nature 1970, 228, 726-734.

(C) 2012 by the authors; licensee MDPI, Basel, Switzerland. This article is an open access article distributed under the terms and conditions of the Creative Commons Attribution license (http://creativecommons.org/licenses/by/3.0/). 